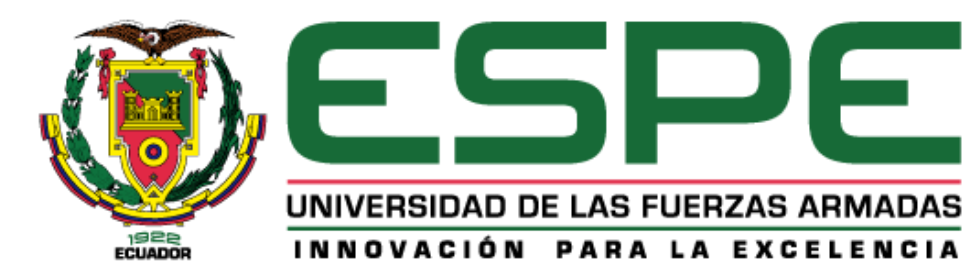

\author{
DEPARTAMENTO DE ELÉCTRICA Y ELECTRÓNICA \\ CARRERA DE INGENIERÍA EN ELECTRÓNICA E INSTRUMENTACIÓN
}

ARTÍCULO ACADÉMICO PREVIO A LA OBTENCIÓN DEL TÍTULO DE INGENIERO EN ELECTRÓNICA E INSTRUMENTACIÓN

TEMA: DETECTION OF THYROID NODULES THROUGH NEURAL NETWORKS AND PROCESSING OF ECHOGRAPHIC IMAGES

AUTORES: Haro Fierro Alex Rubén

Toalombo Toapaxi Julio César

TUTOR: Ing. Galarza Zambrano Eddie Egberto

Latacunga

Agosto, 2020 


\title{
Detection of Thyroid Nodules Through Neural Networks and Processing of Echographic Images
}

\author{
Alex R. Haro ${ }^{1[0000-0001-5245-7334]}$, Julio C. Toalombo ${ }^{1[0000-0002-4577-7548]}$, Eddie E. Galar- \\ $\mathrm{za}^{1[0000-0001-6121-7092]}$ and Nancy E. Guerrón ${ }^{\text {[0000-0003-1971-4647] }}$ \\ ${ }^{1}$ Universidad de las Fuerzas Armadas ESPE, Sangolquí 170501, Ecuador \\ \{arharo, jctoalombo, eegalarza, neguerron\} @espe.edu.ec
}

\begin{abstract}
The abnormal functioning of hormones produces the appearance of malformations in human bodies that must be detected early. In this manuscript, two proposals are presented for the identification of thyroid nodules in ultrasound images, using convolutional neural networks. For the network training, 400 images obtained from a medical center and stored in a database have been used. Free access software (Python and TensorFlow) has been used as part of the algorithm development, following the stages of image preprocessing, network training, filtering and layer construction. Results graphically present the incidence of people suffering from this health problem. In addition, based on the respective tests, it is identified that the system developed in Python has greater precision and accuracy, $90 \%$ and $81 \%$ respectively, than TensorFlow design. Through neural networks, the recognition up to $4 \mathrm{~mm}$ thyroid nodules is evidenced.
\end{abstract}

Keywords: Computer Vision, Convolutional Neural Networks, Thyroid Nodule, Ultrasound Image.

\section{$1 \quad$ Introduction}

The development of technology and engineering techniques have allowed the dissemination of knowledge in all fields of science [1-5]. Its application in medicine has increased with the aim of improving human health, in the treatment of diseases, making prostheses, developing rehabilitation sessions, during surgeries or in the resuscitation of patients [6-9]. Interesting proposals have been generated using informatics, instrumentation, robotics, automation, image and signal processing, among others [10-14]. Image processing as a tool for information and computation is the basis of a growing variety of applications including medical diagnosis, remote sensing, space exploration, computer vision, etc. [15].

As part of medical examinations, medical imaging procedures are performed such as: ultrasound, radiography, tomography, Magnetic Resonance Imaging (MRI), etc. $[16,17]$. Imaging has become an essential component in many fields of medical research as well as in laboratory and clinical practice. Radiologists identify and quantify tumors and skeleton scans on MRI and computed tomography (CT) scans [18]. Neu- 
roscientists detect regional metabolic brain activity from positron emission tomography (PET) and functional MRIs [19]. Until recently, 3D image viewing and quantitative analysis could only be performed using expensive workstations and custom software [20]. Today, much of the visualization and analysis can be done on a budget desktop computer. After receiving an initial medical evaluation, tests are carried out to determine the disease and establish an adequate diagnosis and treatment.

Ultrasonography can evaluate the appearance of the thyroid, a gland located in the neck that regulates the human metabolism [21, 22]. However, metabolism does not always work correctly. Thus, Thyroid nodules can be defined as an abnormal development in thyroid cells forming an internal tumor. Despite most are benign, other have treatment when they are identified in early stages of cancer. Although in terminal phases, doctors cannot assure that a treatment can combat this health problem, causing the patient death [23]. As can be seen in [24], the incidence of thyroid cancer has increased in adults over 65 years, demonstrating that the proper use of ultrasound contributes as a tool for its early detection. In Ecuador, approximately $10 \%$ of the adult population has thyroid nodules, 8 to $12 \%$ of them are cancerous, and therefore the use of technological tools for their early detection are of great relevance.

The purpose of this research is to prepare two proposals that allow detection of thyroid nodules in ultrasound using image processing. Through neural networks, segmentation and classification tasks are carried out to better break down the information for its respective analysis. Two user interfaces are created where results obtained from samples entered are presented to the user. It reduces the generation of false positive results.

The article is organized as follows: in Sect. 1 the introduction and in Sect. 2 the materials and methods. In Sect. 3 the implementation of the proposal is described and in Sect. 4 tests and results obtained. Finally, the conclusions and future work are presented in Sect. 5.

\section{Materials and Methods}

Specialist performs the ultrasound on a patient, then images obtained are analyzed visually and virtually for the detection of nodules. Each image is stored in a database (DB) and will be used to carry out the preprocessing (only in Python) and subsequent treatment of this information. Fig. 1 presents a general diagram of all phases.

Once the preprocessing has been done, detection of nodules is carried out by using a convolutional neural network (CNN) strategy that mimics the visual cortex of the human eye to identify objects. The better the capacity of the computer, the better functionality is obtained. 


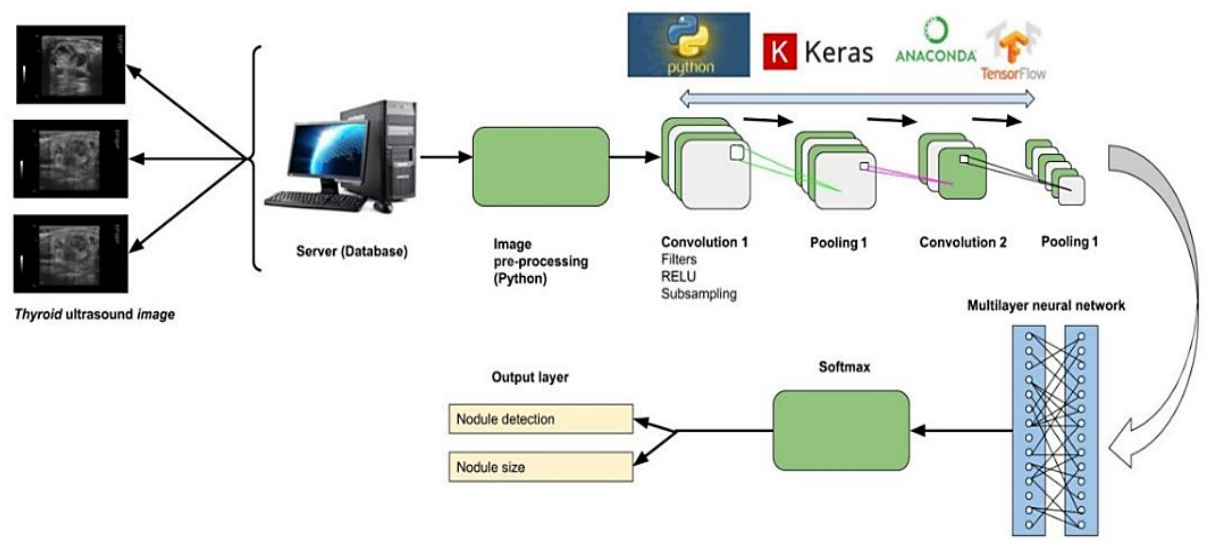

Fig. 1. System general scheme.

\subsection{Definition of Requirements and Parameters}

Neural network development requires the use of Python 2 or 3.7 software and TensorFlow configured and installed correctly. Subsequently, the import of images stored in the DB consisting of 500 ultrasounds captured in a period of 12 months is performed. 400 images are used for network training and 100 for experimental tests. It must be considered the same directory of the images in order to be able to execute in the code.

Regarding the parameters, it must specified the number of times the data set will be repeated during training (iteration $=20$ ); image size, height $=100$ pixels and length $=$ 100 pixels; the number of images, in this case the processing is carried out one by one; preprocessing steps, refers to the number of times the information will be processed (steps $=1000$ ); validation, allows at the end of each iteration to execute a certain number of steps to visualize the learning of the algorithm (validation $=200$ steps); and the convolution filters A and B (filter $A=32$ and filter $B=64$ ) shown in Fig. 2 which are small squares that help to detect shapes by relating them to the pixels in the image.

\subsection{Preprocessing}

The preprocessing stage consist in resize every ultrasound image given from DB, i.e., since the image comes from 0 to 255 pixels, it must be rescaled to an interval between 0 and $1(1 / 255)$ in order to make a more efficient network training. The ultrasound images will be varied, since it can be a man, woman, boy or girl. For this reason, it is necessary to generate an image that has displacement (equal to 0.3), being able to detect the nodules in different positions. When validating, it can be obtained the same image as at the beginning, but now ready to be analyzed in the CNN. This preprocessing is only developed in Python while TensorFlow does it directly. 


\section{Proposal Implementation}

\subsection{Software}

SPYDER IDE must have been configured for the algorithm development in Python. It allows creating scientific packages and is available for Windows, Linux and MacOS. TensorFlow was operated as an open source platform to express machine learning algorithms and Anaconda Navigator is used as a graphical user interface (GUI), to easily start and manage packages, environments and command channels. JupyterLab was used for the development environment due to its excellent flexibility in autonomous learning tasks. Keras took advantage of TensorFlow's high-level API (Application Programming Interface) that was applied to build and train learning models.

\subsection{Neural Network Development}

The preprocessed image (Python case) is used in the neural network to develop its training through the IDE. Neural networks are mathematically represented in (1). Where: $x_{i}=$ input data; $w_{i}=$ synaptic weights; $w_{0}=\mathrm{b}$ is the polarization factor and $m=$ number of filters in layer $n-1$. The result $(r)$ is obtained with the processing of the binary function, i.e., the value of zero or one. In (2) the one and only output generated from the sum between the input vector $x=\left(x_{i}, \ldots, x_{m}\right)$ and the weight vector $w=\left(w_{i}, \ldots, w_{m}\right)$ is presented.

CNNs consist of three fundamental layers which are: input, hidden and output layers. The image is entered (input layer) to extract each of the pixels of the image by applying the different processing phases (hidden layers) and then results are obtained (output layer). Based on them, the thyroid status analysis can be run to determine the presence of nodules and their size. Results are shown in a graphical interface for each software. Mathematical representation of the convolution performed on the network is described in (3).

$$
\begin{gathered}
r=\sum_{i=1}^{m} x_{i} w_{i}+b \\
y=\gamma\left(\sum_{i=1}^{m} x_{i} w_{i}+w_{0}\right) \\
n e t_{j}{ }^{n}=\sum_{k=1}^{K} x_{k}{ }^{n-1} *_{k j}{ }^{n}+b^{n}
\end{gathered}
$$

Figure 2 shows the general scheme of a CNN. It consists of 6 several stages: 1) The selection of the preprocessed image described above; 2) The convolution stage. There 
are two types of convolution: convolution A generally detects contrasts, lines, color changes while convolution B detects more outlined elements as image forms.

3) The pooling 1 stage. It reduces the number of parameters selected with the most common feature of the image, in order to avoid over-fitting, the model. 4) The total connectivity stages. There are the output characteristics that connect to 5) the pooling 2 stage. Characteristics contain trainable connections previously developed in the adaptive programming algorithm on CNN. Finally, in 6) the binary classification stage, results generated after processing are obtained. It details whether there is the presence of thyroid problems in the patient.

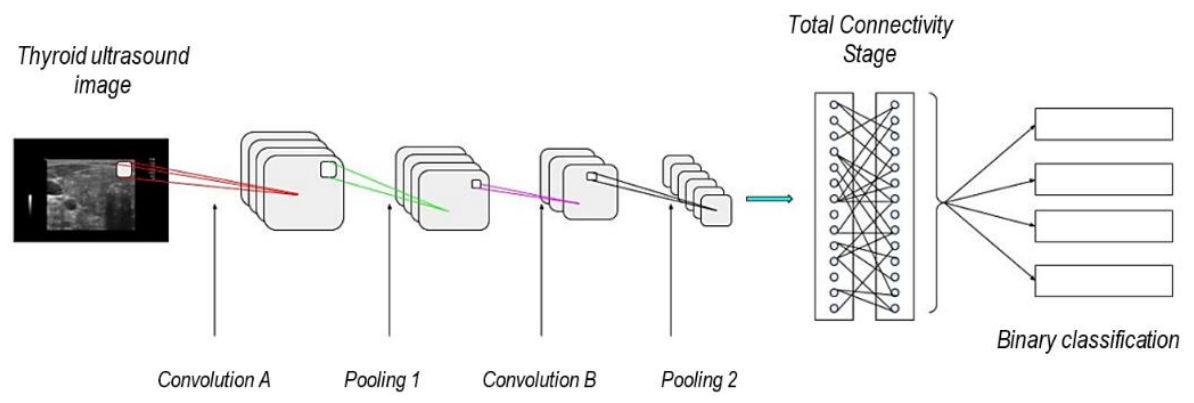

Fig. 2. General scheme of the detection of thyroid nodule by CNN.

\subsection{Neural Network Training}

Once the code is debugged, training and validation begin selecting several images, which will be rescaled to pixels and to all the stages previously described. CNN will be automatically learning. Then the trained network is stored in default format, so that it can be used at any time without having to train it again. The training quality is linked to the size of the database, i.e., the more images are presented to the network, the better it will be for its training and learning parameters.

\subsection{Layer Construction}

In TensorFlow, the layer construction stage is considered as the fundamental part for neural networks. They are layers that extract information from database to solve problems. First layer performs the transformation of the image format to a twodimensional arrangement, keeping in mind that this layer has no parameters to learn. Next two layers are connected to each other, the first providing 128 nodes and the other with 10 nodes which allows network learning. Subsequently, the database consisting of 400 grayscale ultrasound images of thyroid with 255 pixels is loaded. 


\subsection{Image Processing}

In TensorFlow, image processing is done while $\mathrm{CNN}$ is running and as the training is done, values of the Kernel parameters, filters, their learning and activation are adjusted. All these values are saved and are also transparent.

Kernel. Kernel parameters or weights are the values of a matrix that has different resignations depending on each $\mathrm{CNN}$. This is a great advantage at the time of the learning process since each Kernel has a reduced size. An example may be having a $3 \times 3$ matrix; there are only 9 parameters that must be adjusted and having 32 filters gives a total of 288 parameters.

$$
h * f(u, v)=\sum_{x}^{n} \sum_{y}^{n} h(x, y) * f(x-u, y-v)
$$

Figure 3 shows the convolution performed between an image and a Kernel matrix. If Kernel is an $\mathrm{h}(\mathrm{x})$ of dimensions' $n \times n$ and the object matrix $f(x, y)$ is of size $n x n$, using the definition of the $2 \mathrm{D}$ convolution for a point of the resulting matrix, we obtain (4).

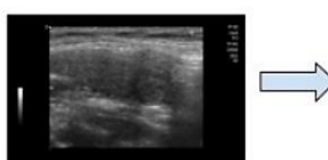

Image

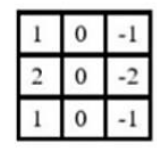

Kernel

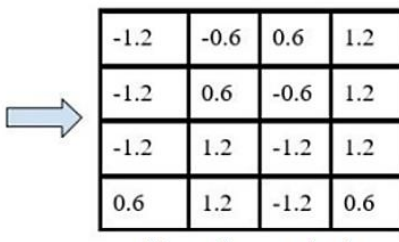

Kernel convolution

Fig. 3. The image performs a convolution with a Kernel.

It is easy to see that to calculate the convolution of a single point $(u, v)$ of the matrix resulting from the convolution, $n^{2}$ products are required, as should be done for each of the $N^{2}$ elements of $f(x, y)$. In total, $n^{2} x N^{2}$ is required.

Filters. First filter is responsible for eliminating noise to have a clean image. It eliminates excess brightness, reduces lighting and shapes that alter the image, etc. Second filter is in charge of detecting similarities of circular shapes and making the relation of the area with the pixels, to determine the size of the nodule. The third is a classifier filter that, with data from previous ones, agrees to classify the image in which there are nodules.

Learning and Activation. Learning parameters are the system reliability, they are measured in percentage. Recommended values for these parameters must be at least 
$70 \%$, considering that these values increase the more the network is trained. Activation parameters allow CNN to be activated and deactivated appropriately and are very significant for testing and exposing the results obtained.

\subsection{Interface Development}

To expose the results to the user, an interface is designed with the objective of showing clearly what $\mathrm{CNN}$ identified. For this, a main window is used that contains a Frame where all the widgets of the interface are organized. Python contains several libraries for GUI development, these are: Tkinter, WxPython, PyQT, PyGTk, Matplotlib, etc. In this case, Tkinter library was used to allow Python to be linked with the TCL/TK library, Python GUI is shown in Fig. 4. On the other hand, TensorFlow uses Anaconda Navigator for the development of the GUI that, through the execution of Jupyter Notebook, two programs are created: 1) For network training and 2) To show the results obtained as shown in Fig. 5.

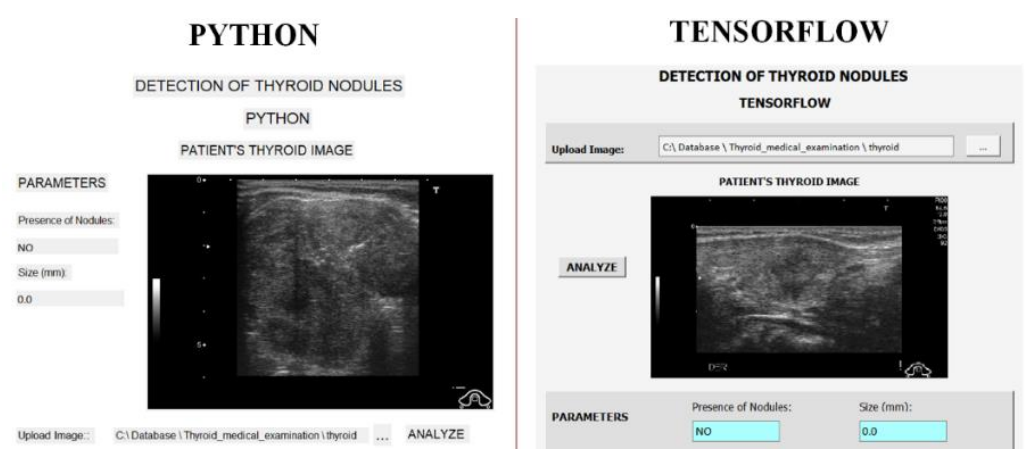

Fig. 4. Graphical User Interface for each software used.

\section{Experimental Results}

Due to the visual and technological analysis of the specialist, it was possible to separate cases with nodular presence both by age and gender. Cases with the presence of thyroid nodules are encapsulated in age ranges for classification into: young people ( $<24$ years old), adults (25-59 years old) and older adults (>60 years old). With a lower percentage of $3.6 \%$ they occur in young population. This inclination is due to cases that are hereditary and no longer provoked. The appearance in the adult population is increasingly frequent with $44 \%$. This incidence has increased each year due to the population type of diet with iodine deficiency which is the main cause in formation of thyroid nodules. The majority trend occurs in older adults with $52.4 \%$, it is an aggravating factor the low frequency they visit the endocrinologist for preventive control and relevant examinations. These trends can be seen reflected in Fig. 5. 


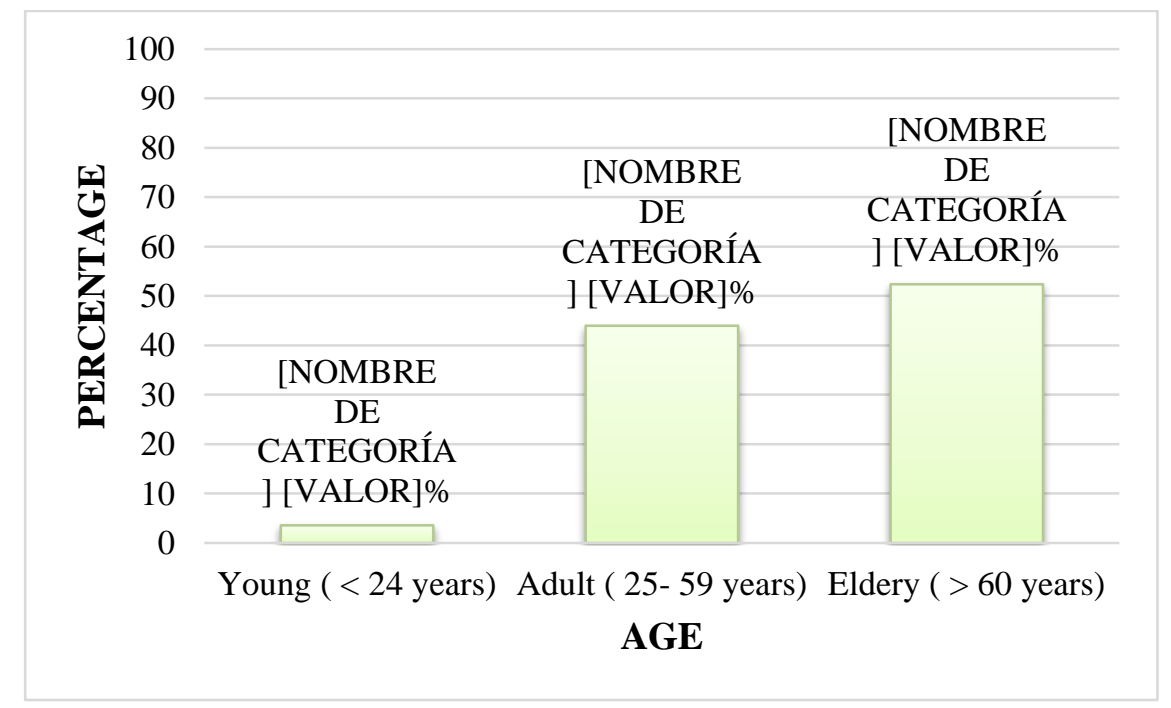

Fig. 5. Percentage of presence of thyroid nodules according to age range.

Another important categorization to analyze the presence of malformations in the thyroid is according to gender. Men with just $10.8 \%$, is understandable since men are hormonally more stable. In women with $89.2 \%$ the trend is predominant, since it is more prone to hormonal imbalance. These values are shown in Fig. 6. It should be clarified that these global data (by age and by gender) have already been defined by the specialist since patients do not allow to show specific data regarding the ultrasounds obtained.

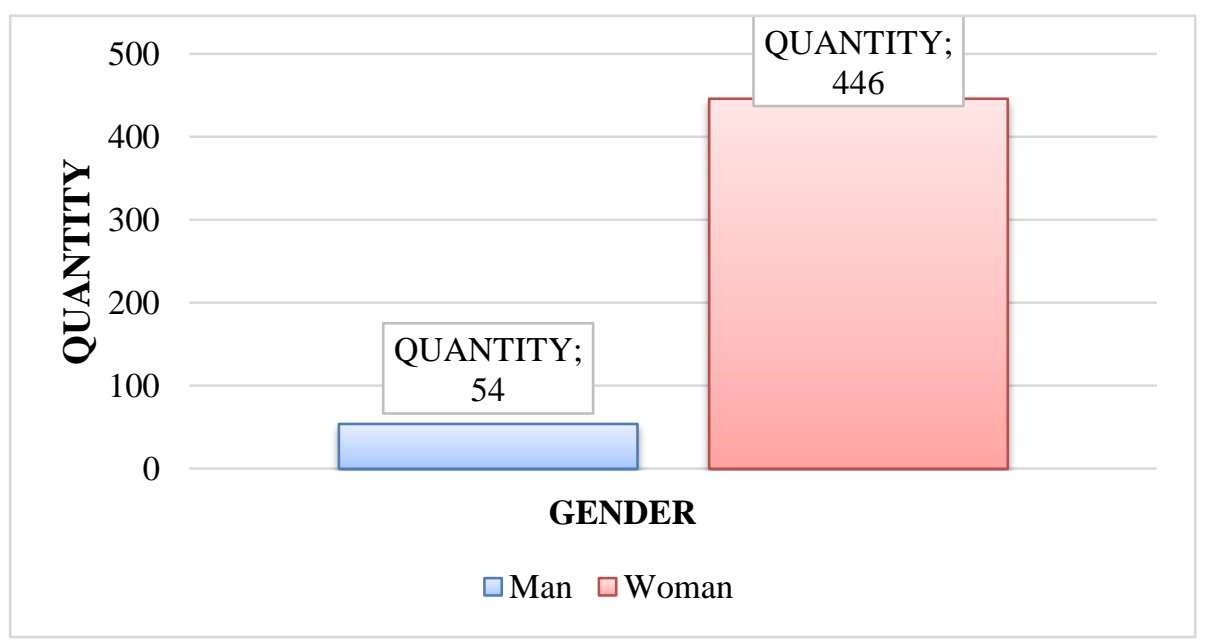

Fig. 6. Percentage of presence of thyroid nodules according to gender. 
There are only two types of thyroid nodules that people can have: malignant and benign. The size of a nodule indicates its degree of danger, $10 \mathrm{~mm}$ being the most desirable for early treatment. For its phases determination, three parameters are considered: size (T), spread to nodes (N) and spread to other nearby organs (M). In Fig 7. several thyroid nodules are detected in each software evidencing differences when showing its size. It allows authors to determine an accuracy test per software to define which one is the best option as a complementary tool for specialists.

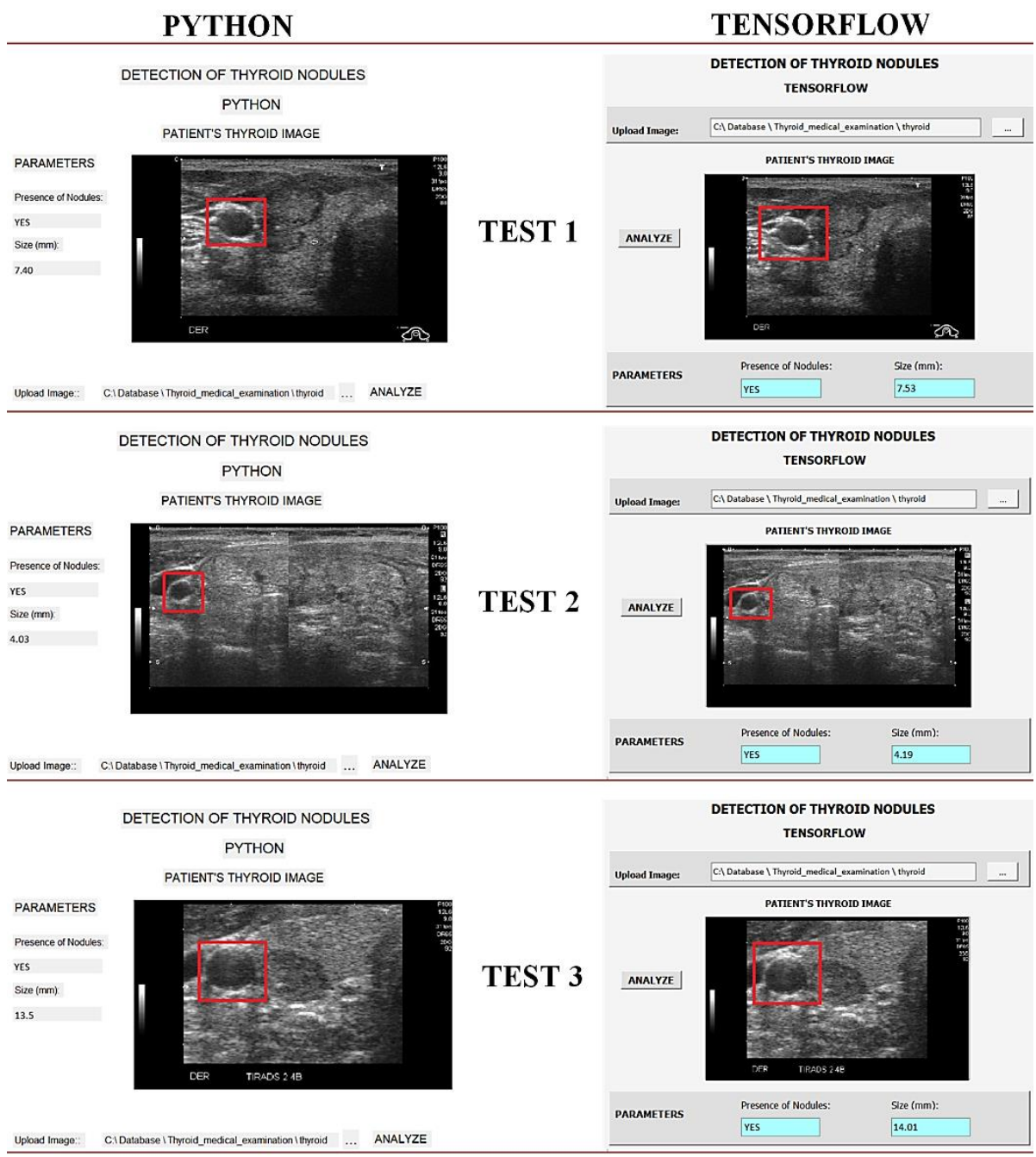

Fig. 7. Thyroid nodule detection per software. 


\subsection{Python Tests}

To obtain a correct validation, sensitivity (SENS), specificity (ESP) and an external validity corresponding to the positive predictive value (PPV) and negative predictive value (NPV) must be analyzed. This analysis is performed to demonstrate the tool ability to detect the presence and absence of thyroid nodules. For the set of tests carried out (100 images) Python tool gave the following results as shown in Table 1.

Table 1. Test results from Python with 100 images (True diagnosis)

\begin{tabular}{llll}
\hline Results & Presence & Absence & Total \\
\hline True Positive & 50 & 4 & 54 \\
True Negative & 2 & 44 & 46 \\
Total & 52 & 48 & 100 \\
\hline
\end{tabular}

The programming algorithm correctly classified 50 from 52 images containing thyroid nodules, obtaining a SENS of $96.15 \%$. On the other hand, it classified 44 from 48 images with the absence of thyroid nodules, obtaining an ESP of 91.67\%. The SENS and ESP are obtained by (5) and (6).

$$
\begin{aligned}
\mathrm{SENS} & =\frac{\mathrm{TP}}{\mathrm{TP}+\mathrm{FN}} * 100 \% \\
\mathrm{ESP} & =\frac{\mathrm{TN}}{\mathrm{TN}+\mathrm{FP}} * 100 \%
\end{aligned}
$$

Where:

TP: True Positive

TN: True Negative

FP: False Positive

FN: False Negative

To obtain PPV and NPV, data is analyzed horizontally. In this way, the probability is obtained that the patient does or does not have thyroid nodules. To calculate these values, it should be followed the expressions:

$$
\begin{aligned}
\mathrm{PPV} & =\frac{\mathrm{TP}}{\mathrm{TP}+\mathrm{FP}} * 100 \% \\
\mathrm{NPV} & =\frac{\mathrm{TN}}{\mathrm{TN}+\mathrm{FN}} * 100 \%
\end{aligned}
$$

The probability that a patient with positive results (PPV) complies with the pathology is $92.59 \%$ and the probability of not containing the pathology when the test result is negative (NPV) is $95.65 \%$. 


\subsection{TensorFlow Tests}

Table 2 shows the results with respect to TensorFlow, which correctly classified 49 images from the 52 that contained thyroid nodules, it allows to obtain a SENS of $94.23 \%$, and from 48 images, the algorithm classified 42 with the absence of thyroid nodules, obtaining an ESP of $87.50 \%$. Values in this analysis are substantiated by using recursively equations (5-8).

Table 2. Test results from TensorFlow with 100 images (True diagnosis)

\begin{tabular}{llll}
\hline Results & Presence & Absence & Total \\
\hline True Positive & 49 & 6 & 55 \\
True Negative & 3 & 42 & 45 \\
Total & 52 & 48 & 100 \\
\hline
\end{tabular}

Examining the obtained calculations, it can be mentioned that TensorFlow has a $94.23 \%$ of SENS to detect thyroid nodules. Additionally, it has an $87.50 \%$ of ESP to correctly classify patients who do not have nodules. It is also important to mention that the probability that a patient with positive results has the pathology (PPV) is $89.09 \%$ and the probability of not containing the pathology when the test result is negative (NPV) is $93.33 \%$.

\section{$5 \quad$ Conclusions and Future Work}

Within the diagnosis of thyroid nodules, the pathological phase consists of the analysis of the extracted tissue and the clinical phase consists of the execution of a medical examination and an imaging study. The system has been designed to be able to identify nodules up to $4 \mathrm{~mm}$ in size, these can be malignant or benign. When a nodule is detected, it must be followed up and analyzed to determine if it is malignant. Antecedents that can lead to a malignant nodule are: rapid growth of the nodule, sudden and recent change in voice, difficulty swallowing or breathing, palpation of the nodule as a hard mass or enlarged neck nodes.

Women are more likely to have this pathology compared to men due to the existing variation in hormonal stability caused by two factors: 1) hormonal disturbances and 2) iodine deficiency in daily diet. Similarly, it has been identified that older adults have a higher incidence of suffering from these hormonal problems.

In this application, Python is the programming tool that offers greater reliability with a sensitivity of $96.15 \%$ and a specificity of $91.67 \%$. TensorFlow reach less values, $94.23 \%$ of sensitivity and $87.50 \%$ for specificity. However, it should be considered that the application was developed with only 500 images, 100 of them were destined for tests this investigation, so if the $\mathrm{CNN}$ is trained with a larger database, the result may vary. The objective of this research is not to establish a direct diagnosis in patients, but to provide a useful tool for specialists to allow them to determine with 
greater certainty the presence of thyroid nodules and additionally to provide approximate information on their size.

Applications developed with TensorFlow that use CNN require immense DB to improve its training and provide higher quality results. This is why authors of this document establish this particular as a proposal for future work. Additionally, a greater number of filters could be implemented, since in the development of this investigation a notable improvement was evident when increasing them. Another improvement which should be incorporated is the detection of the number of nodules present in the echography. To accomplish this, image processing should be improved.

Acknowledgments. The authors thank the Universidad de las Fuerzas Armadas ESPE for the approval of the research project entitled: "Redes neuronales para la detección de nódulos de tiroides mediante procesamiento de imágenes de ecografías" for supporting the development of this work and especially to Jorge Buele and Esteban X. Castellanos.

\section{References}

1. Brodski-Guerniero, A., Paasch, G.F., Wollstadt, P., Özdemir, I., Lizier, J.T., Wibral, M.: Information-Theoretic Evidence for Predictive Coding in the Face-Processing System. J. Neurosci. (2017). https://doi.org/10.1523/JNEUROSCI.0614-17.2017.

2. García, C.A., Buele, J., Espinoza, J., Castellanos, E.X., Beltrán, C., Pilatasig, M., Galarza, E., García, M. V.: Fuzzy control implementation in low cost CPPS devices. In: IEEE International Conference on Multisensor Fusion and Integration for Intelligent Systems. pp. 162-167 (2017). https://doi.org/10.1109/MFI.2017.8170423.

3. Petersen, F., Brown, A., Pather, S., Tucker, W.D.: Challenges for the adoption of ICT for diabetes self-management in South Africa. Electron. J. Inf. Syst. Dev. Ctries. (2019). https://doi.org/10.1002/isd2.12113.

4. Buele, J., Espinoza, J., Pilatásig, M., Silva, F., Chuquitarco, A., Tigse, J., Espinosa, J., Guerrero, L.: Interactive system for monitoring and control of a flow station using labVIEW. In: Advances in Intelligent Systems and Computing. pp. 583-592 (2018). https://doi.org/10.1007/978-3-319-73450-7_55.

5. Chen, G.: Research on fuel supply intelligent monitoring system design based on PLC. J. Comput. Theor. Nanosci. (2017). https://doi.org/10.1166/jctn.2017.6932.

6. Buele, J., Varela-Aldás, J., Salazar, F.W., Soria, A., Andaluz, V.H.: Wheelchair Controlled by Eye Movement Using Raspberry Pi for ALS Patients. In: Advances in Intelligent Systems and Computing. pp. 124-136 (2020). https://doi.org/10.1007/9783-030-35740-5_9.

7. Albiol-Pérez, S., Palacios-Navarro, G., Guerrón-Paredes, N., Gil-Gómez, J.A., Quilis, J.A.L., Gil-Gómez, H., Manzano, P.: The perfetti method, a novel virtual fine motor rehabilitation system for chronic acquired brain injury. In: Proceedings - REHAB 2014 (2014). https://doi.org/10.4108/icst.pervasivehealth.2014.255251.

8. Andrea Sánchez, Z., Santiago Alvarez, T., Roberto Segura, F., TomásÂ Núñez, C., Urrutia-Urrutia, P., Franklin Salazar, L., Altamirano, S., Buele, J.: Virtual 
Rehabilitation System Using Electromyographic Sensors for Strengthening Upper Extremities. In: Smart Innovation, Systems and Technologies. pp. 231-241 (2020). https://doi.org/10.1007/978-981-13-9155-2_19.

9. Galarza, E.E., Pilatasig, M., Galarza, E.D., López, V.M., Zambrano, P.A., Buele, J., Espinoza, J.: Virtual reality system for children lower limb strengthening with the use of electromyographic sensors. In: Lecture Notes in Computer Science (including subseries Lecture Notes in Artificial Intelligence and Lecture Notes in Bioinformatics). pp. 215-225 (2018). https://doi.org/10.1007/978-3-030-03801-4_20.

10. Rybarczyk, Y., Deters, J.K., Gonzalvo, A.A., Gonzalez, M., Villarreal, S., Esparza, D.: ePHoRt project: A web-based platform for home motor rehabilitation. In: Advances in Intelligent Systems and Computing (2017). https://doi.org/10.1007/978-3-319-565385_62.

11. Quezada, A., Juárez-Ramírez, R., Jiménez, S., Noriega, A.R., Inzunza, S.: An empirical study on usability operations for autistic children. In: Advances in Intelligent Systems and Computing (2017). https://doi.org/10.1007/978-3-319-56538-5_64.

12. Salazar, F.W., Núñez, F., Buele, J., Jordán, E.P., Barberán, J.: Design of an Ergonomic Prototype for Physical Rehabilitation of People with Paraplegia. In: Advances in Intelligent Systems and Computing. pp. 341-353. Springer, Cham (2020). https://doi.org/10.1007/978-3-030-33614-1_23.

13. Pilatásig, M., Tigse, J., Chuquitarco, A., Pilatásig, P., Pruna, E., Acurio, A., Buele, J., Escobar, I.: Interactive system for hands and wrist rehabilitation. In: Advances in Intelligent Systems and Computing. pp. 593-601. Springer Verlag (2018). https://doi.org/10.1007/978-3-319-73450-7_56.

14. Buele, J., López, V.M., Franklin Salazar, L., Edisson, J.H., Reinoso, C., Carrillo, S., Soria, A., Andrango, R., Urrutia-Urrutia, P.: Interactive System to Improve the Skills of Children with Dyslexia: A Preliminary Study. In: Smart Innovation, Systems and Technologies. pp. 439-449 (2020). https://doi.org/10.1007/978-981-13-9155-2_35.

15. Fernández-S, Á., Salazar-L, F., Jurado, M., Castellanos, E.X., Moreno-P, R., Buele, J.: Electronic system for the detection of chicken eggs suitable for incubation through image processing. In: Advances in Intelligent Systems and Computing. pp. 208-218 (2019). https://doi.org/10.1007/978-3-030-16184-2_21.

16. Spaide, R.F., Fujimoto, J.G., Waheed, N.K., Sadda, S.R., Staurenghi, G.: Optical coherence tomography angiography, (2018). https://doi.org/10.1016/j.preteyeres.2017.11.003.

17. Koo, T.K., Silvia, N.: Actuator-Assisted Calibration of Freehand 3D Ultrasound $\begin{array}{llllll}\text { System. J. Healthc. } & \text { Eng. 2018, } 9314626 \quad \text { (2018). }\end{array}$ https://doi.org/10.1155/2018/9314626.

18. Debette, S., Schilling, S., Duperron, M.G., Larsson, S.C., Markus, H.S.: Clinical Significance of Magnetic Resonance Imaging Markers of Vascular Brain Injury: A Systematic Review and Meta-analysis. JAMA Neurol. (2019). https://doi.org/10.1001/jamaneurol.2018.3122.

19. T. Thomas, H.M., Devakumar, D., Sasidharan, B., Bowen, S.R., Heck, D.K., James Jebaseelan Samuel, E.: Hybrid positron emission tomography segmentation of heterogeneous lung tumors using 3D Slicer: improved GrowCut algorithm with threshold initialization. J. Med. Imaging. (2017). 
https://doi.org/10.1117/1.jmi.4.1.011009.

20. Dou, Q., Yu, L., Chen, H., Jin, Y., Yang, X., Qin, J., Heng, P.A.: 3D deeply supervised network for automated segmentation of volumetric medical images. Med. Image Anal. 41, 40-54 (2017). https://doi.org/10.1016/j.media.2017.05.001.

21. Dighe, M., Barr, R., Bojunga, J., Cantisani, V., Chammas, M.C., Cosgrove, D., Cui, X.W., Dong, Y., Fenner, F., Radzina, M., Vinayak, S., Xu, J.M., Dietrich, C.F.: Thyroid ultrasound: State of the art part 1 - Thyroid ultrasound reporting and diffuse thyroid diseases, (2017). https://doi.org/10.11152/mu-980.

22. Chi, J., Walia, E., Babyn, P., Wang, J., Groot, G., Eramian, M.: Thyroid Nodule Classification in Ultrasound Images by Fine-Tuning Deep Convolutional Neural Network. J. Digit. Imaging. (2017). https://doi.org/10.1007/s10278-017-9997-y.

23. Mazzaglia, P.J., Muraveika, L.: Normal thyroid appearance and anatomie landmarks in neck ultrasound. In: Advanced Thyroid and Parathyroid Ultrasound. pp. 77-86 (2017). https://doi.org/10.1007/978-3-319-44100-9_9.

24. Haymart, M.R., Banerjee, M., Reyes-Gastelum, D., Caoili, E., Norton, E.C.: Thyroid Ultrasound and the Increase in Diagnosis of Low-Risk Thyroid Cancer. J. Clin. Endocrinol. Metab. 104, 785-792 (2018). https://doi.org/10.1210/jc.2018-01933. 